\title{
Structurally Integrated Joint Torque Sensors in an Assistive Upper Limb Exoskeleton
}

\author{
Markus Hessinger ${ }^{1}$, Johannes Krenzer ${ }^{1}$, Eike Christmann ${ }^{1}$, Jürgen Hielscher ${ }^{1}$, Mario Kupnik ${ }^{1}$, Roland \\ Werthschützky ${ }^{1}$ \\ ${ }^{1}$ Institute of Electromechanical Design, Measurement and Sensor Technology Group, Technische \\ Universität Darmstadt, Merckstr. 25, 64283 Darmstadt, Germany \\ Corresponding author: m.hessinger@emk.tu-darmstadt.de
}

\begin{abstract}
:
This paper describes the design process of a torque sensor, which is structurally integrated into the kinematic chain of an assistive upper limb exoskeleton to measure user interaction. Two different approaches of torque sensor integration are discussed regarding the nominal torque loads on each joint and applied transverse stresses. The final sensor concept consists of an aluminum spoke wheel deformation element with four strain gauges connected to a Wheatstone bridge. It is mounted between the motor casing and gear drive of each joint to measure the torque of the actuator and user. A sensor electronics board including signal processing and an EtherCAT interface is integrated in each joint. The torque sensor shows linear transmission characteristics for $0.4 \mathrm{Nm}$ nominal rated torque with a linear error of $0.51 \%$ and a hysteresis error of $0.53 \%$.
\end{abstract}

Key words: torque sensor, structurally integrated, assistive exoskeleton, user interaction, sensor electronics

\section{Introduction}

Ambient Assisted Living (AAL) comprises a set of methods, concepts and systems to support elderly and physically handicapped people to independently manage their activities of daily living [1]. The range of AAL solutions vary from software platforms to electromechanical systems, which assist users in complex movements of the upper and lower limbs. Here, an assistive upper limb exoskeleton (Fig. 1) is developed to provide haptic guidance during positioning tasks of the hand and arm. In order to recognize the movement intention or situation of the user without the need of additional bioelectrical signals, such as electromyography (EMG), torque sensors are integrated into the joints of the exoskeleton. Thus, derivations in the measured torque signals to the expected torque signals are interpreted as a user input.

Various approaches to recognize the user's movement intention are applied in research and commercial products for assistive robotic systems. For exoskeletons, interaction force, torque, EMG measurements are used to generate desired movement trajectories for the user (Tab. 1). Since precise motion estimation based on EMG signals is difficult to realize [2], a system for user intention recognition based on structurally integrated torque sensors is proposed in this work.

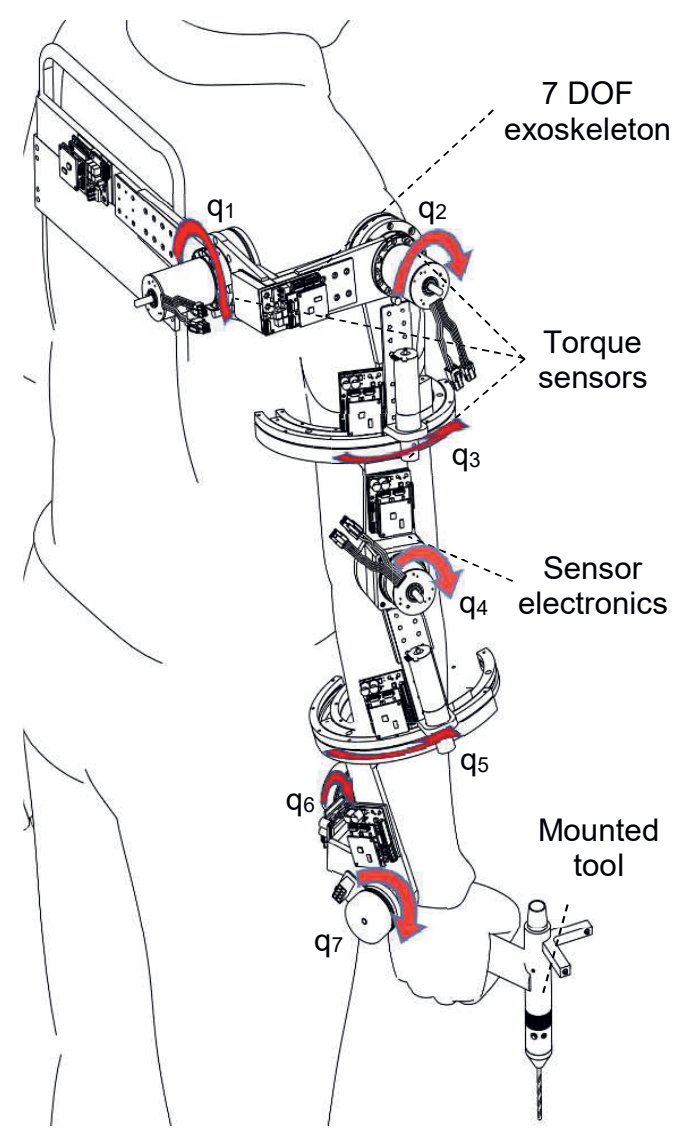

Fig. 1: Assistive upper limb exoskeleton design with seven actuated degrees of freedom (DOF) $q_{1} \ldots q_{7}$. 
Tab. 1: Classification of upper limb exoskeletons

\begin{tabular}{ccccc}
\hline Name, Reference & Joints & Application & DOF & Main control inputs \\
\hline [2] & Shoulder, elbow, wrist & Power assistance & 7 & sEMG, Torque/ Force \\
{$[3]$} & Shoulder, elbow & Rehabilitation & 4 & Torque/ Force \\
SAM, [4] & Shoulder, elbow, wrist & Power assistance & 7 & Torque/ Force \\
SUEFUL-7, [8] & Shoulder, elbow, wrist & Power assistance & 7 & sEMG, Torque/ Force \\
{$[9]$} & wrist & Power assistance & 1 & EMG, EEG \\
{$[10]$} & Shoulder, elbow, wrist & Rehabilitation & 9 & EMG, Force \\
\hline
\end{tabular}

Several research groups have developed exoskeletons with movement intention recognition based on torque and force sensing [2-4]. Some approaches integrate an external signal processor to filter and preprocess the measured force and torque [3]. Some projects integrate torque or force sensors into the kinematic chain of the exoskeleton [5]. Less common are approaches using integrated sensor electronics $[6,7]$ with an analog-to-digital converter with 16-Bit and an FPGA to process the measured signals. For transmitting sensor and actuator data, a fieldbus communication system is implemented. Therefore, apart of the power supply, only one wire is necessary.

In the following sections, two different sensor design concepts are discussed and a sensor system with a sensor electronics board is evaluated. Finally, a concept for user intention recognition is introduced.

\section{Sensor Integration Concept}

The most common method to measure the load signal on a rotation system is to transform a resulting surface strain with strain gauges, applied to a rotating shaft or deformation element, into an equivalent electrical signal. Since industrial torque sensors do not satisfy size and weight restrictions of the assistive robotic system, a structurally integrated sensor system is investigated. The main challenges in choosing the position of the sensing element in the joint structure of the exoskeleton are stiffness requirements of the kinematical chain. Concerning this, an approach with integrated strain gauges in the joint structure [11] is compared to a method with an additional deformation element [12].

The required nominal strain to measure with a foil strain gauge is $1 \mathrm{~mm} / \mathrm{m}$. Thus, the deformation element, integrated into the rigid links of the exoskeleton, must provide the required strain on the surface depending on the joint torque (Tab. 2).
Tab. 2: Computed maximal joint torques with $20 \mathrm{~N}$ load at the end effector.

\begin{tabular}{cccccccc}
\hline Joint & $\mathrm{q}_{1}$ & $\mathrm{q}_{2}$ & $\mathrm{q}_{3}$ & $\mathrm{q}_{4}$ & $\mathrm{q}_{5}$ & $\mathrm{q}_{6}$ & $\mathrm{q}_{7}$ \\
\hline $\begin{array}{c}\text { Torque } \\
(\mathrm{Nm})\end{array}$ & 29 & 26 & 21 & 15 & 9 & 5 & 2
\end{tabular}

The rigid links of the exoskeleton consist of a combination of carbon fiber reinforced polymer (CFRP) and aluminum alloy. The mechanical power of the BLDC motors is transitioned via Harmonic Drive ${ }^{\circledR}$ precision gears (gear ratio 1:100, efficiency 0.85 ) at each joint. Stress simulations of the link elements show that the required strain on the surface is obtained by weakening the material at certain positions (Fig. 2, left). Since the resulting strain appears on both sides of the element, it is possible to measure the joint torque with a half-bridge configuration (Fig. 2, right). The advantages of this approach is a direct measurement of the joint torque at the link element of the exoskeleton, no extra deformation element is necessary.

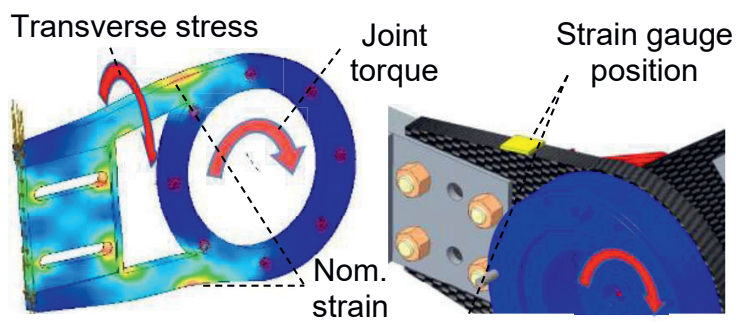

Fig. 2: FEM stress simulation of the joint link with nominal joint torque (left). Concept for torque sensor integration with strain gauges into kinematic structure of the exoskeleton joint axis (right).

Since the mechanical properties of CFRP are depended on the structure of the composite material (mostly unidirectional carbon filament), the E-modulus and strain transmission behavior is analyzed in a four-point bending test rig (Fig. 3). 


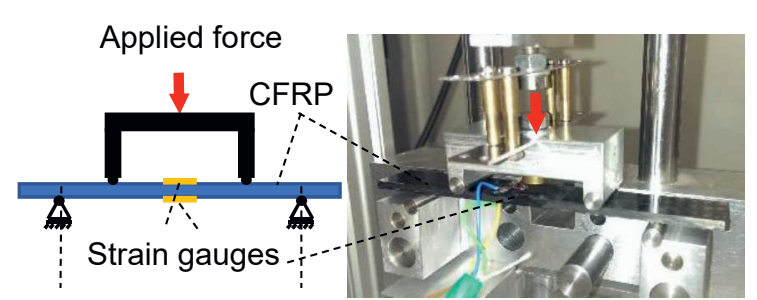

Fig. 3: Strain transmission characteristics test setup for CFRP sample.

The measurement results show an E-modulus of $104 \mathrm{GPa}$ and linear transmission behavior (linearity error $<1 \%$ ) of the CFRP probe.

Next, the transverse torque on each joint is investigated. The weight of the exoskeleton generates a torque perpendicular to the rotational joint axis, which applies transverse stress to the sensing element. The first two shoulder joints suffer from the highest transverse stress (resulting torque $\mathrm{T}_{\mathrm{ts}}\left(\mathrm{q}_{1}\right)=28 \mathrm{Nm}, \mathrm{T}_{\mathrm{ts}}\left(\mathrm{q}_{2}\right)=$ $10,5 \mathrm{Nm})$. Since the structure of the rigid body is weakened for the strain transmission of the sensing element, the transverse stress exceeds the tensile strength of the CFRP part. Thus, direct torque measurement at the joint link with foil strain gauges risks damage to the exoskeleton parts.

As an alternative, it is possible to measure the torque between the actuator and gear drive. This leads to a reduction of the load to the sensor in proportion to the gear ratio (1/100) and a decoupling of the sensing element to the kinematic structure. In order to reduce weight and save space, the sensing element is integrated into the flange of the motor (Fig 4.)

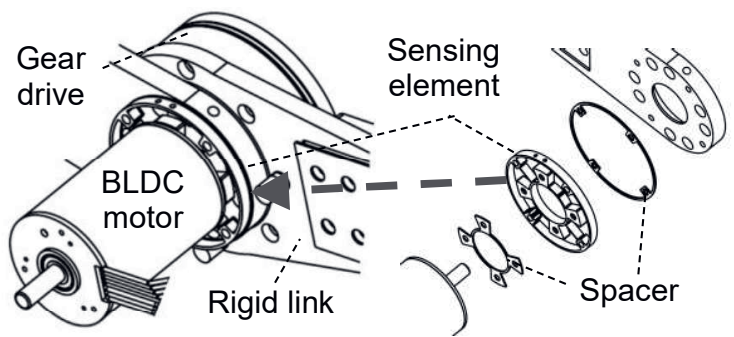

Fig. 4: Concept for torque sensor integration into motor flange of joint axis.

\section{Sensor Design and Calibration}

The geometry of the deformation element is based on a spoke wheel structure to transduce the motor torque into a strain that is measured with four strain gauges. The sensing element is used as a flange to connect the motor with the gear drive. The dimensions of the spoke wheel bridges are designed depending on the applied torques by the motors. The nominal torque of the BLDC motors for the first and second DOF of the shoulder joint is $0.379 \mathrm{Nm}$. With FEM simulations, the deformation element is dimensioned with a bridge size of $4 \times 0.45 \mathrm{~mm}$ (Fig. 5, right).

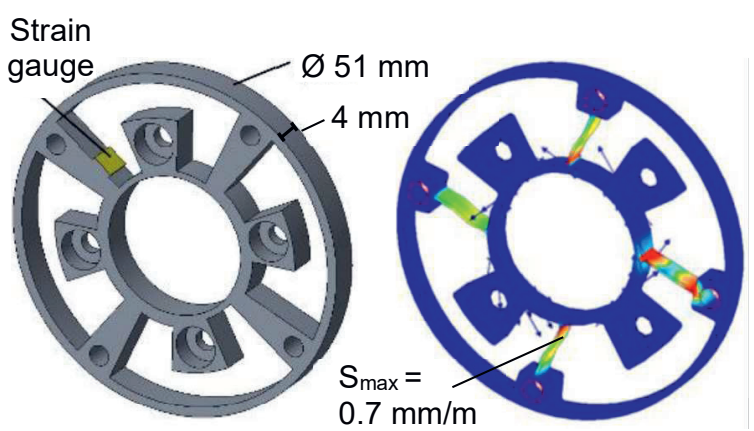

Fig. 5: Deformation element geometry with strain gauge positions (left) and spoke wheel dimensioning with FEM (right).

The deformation element is CNC manufactured using aluminum alloy, polished and cleaned. The dimensions of the bridges allow a simple positioning of the strain gauges, using the edges as landmarks. Four strain gauges (type 1-LA11K3/350-E, Hottinger Baldwin Messtechnik $\mathrm{GmbH}$, Germany) are applied with cyanoacrylate adhesive (type Z70, HBM) and connected to a full bridge circuit. The sensor is calibrated in both directions with weights via a defined lever arm (Fig. 6, left). At first, the sensor is loaded three times with the maximum weight $(0.35 \mathrm{Nm})$. Secondly, the sensor is loaded and unloaded with $50 \mathrm{~g}$ weights in nine steps (Fig. 6, right). The voltage is measured with a 6 -wire bridge amplifier (type EL3356, Beckhoff Automation $\mathrm{GmbH} \&$ Co. KG, Germany).

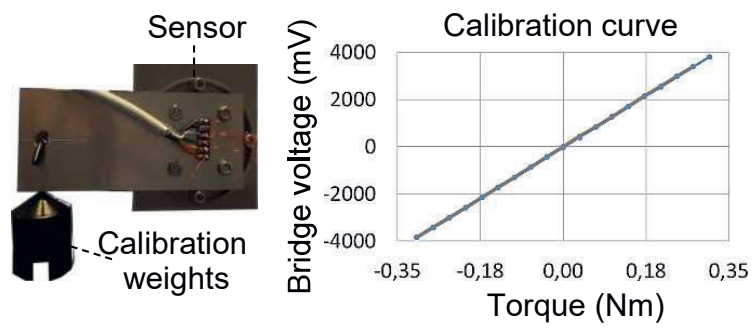

Fig. 6: Torque sensor calibration setup with weights (left) and calibration curve of sensor "shoulder 2" (right).

The sensor shows a linear transmission behavior with only small hysteresis error (Tab. 3).

Tab. 3: Sensor calibration results.

\begin{tabular}{cccc}
\hline Sensor & $\begin{array}{c}\text { Gain } \\
(\mathrm{mV} / \mathrm{Nm})\end{array}$ & $\begin{array}{c}\text { Lin. } \\
\text { error }\end{array}$ & $\begin{array}{c}\text { Hys. } \\
\text { error }\end{array}$ \\
\hline Shoulder 1 & 11800 & 0.012 & 0.021 \\
Shoulder 2 & 12300 & 0.005 & 0.005
\end{tabular}

A reason for the occurring measurement error is a position error due to manual application of the strain gauges. 


\section{Sensor Electronics and System Evaluation}

Strain gauges change their resistance when they are stretched or compressed. The change of resistance is small and requires sensitive circuiting to measure accurately. The Wheatstone bridge is a common circuit to convert the small resistance change into a measurable voltage. Depending on the number of used strain gauges there are different possibilities to wire up a Wheatstone bridge. For the integrated torque sensor, a full-bridge is chosen, providing a high sensitivity and temperature compensation. In contrast to a current source for the excitement supply, using a voltage supply needs no additional hardware. Additionally, the lead resistance is negligible with four-terminal sensing.

The Wheatstone bridge provides a full-scale of $2 \mathrm{mV} / \mathrm{V}$ with $350 \Omega$ strain gauges. With an excitation supply of $5 \mathrm{~V}$, an output of $10 \mathrm{mV}$ is generated. To exploit the scale range of an analog-to-digital converter (ADC), an amplifier is essential. The bridge excitement is used simultaneously as the reference voltage for the analog-to-digital converter (Fig. 7). As a result, any drift in excitation voltage also influences the reference voltage and removes the drift error.

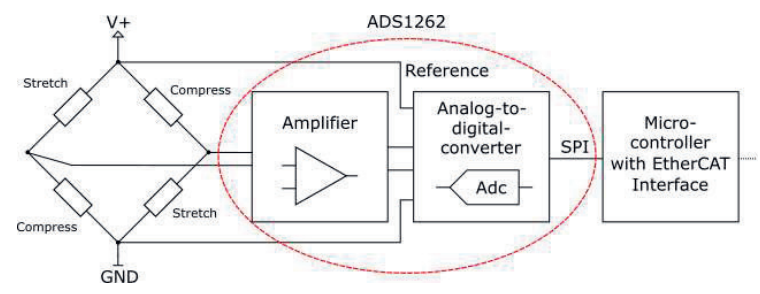

Fig. 7: Measurement chain with signal amplification, $A D C$ and digital signal processing.

For integrated measurement data analysis, a $\Delta \Sigma$ analog-to-digital converter (type ADS1262, Texas Instrument, USA) is used with an integrated PGA with an amplification up to 32 , a resolution of 32-bit and a sampling rate up to 38.4 kSPS. The signal is processed with a microcontroller, providing an EtherCAT interface for signal integration into the global fieldbus of exoskeleton. The complete sensor electronics board is integrated into each joint of the exoskeleton. Apart of the Ethernet connection and wires for the energy supply, no additional wires are necessary. To reduce noise, the length of the lead wire is as short as possible. The leads of the signal, the excitation and the remote sense are twisted. The lead resistance is negligible since the four-terminal sensing is applied.

The weight force of the motor leads to an error ( $\sim 3 \%$ of the nominal torque), depending on the joint angles of the exoskeleton. Since the gravity vector is measured through an IMU at the base of the exoskeleton, the error of the motor weight is cancelled out with signal processing.

The designed sensor board is connected to a motor control stack (type IFM Drive DC 300, Core C22, COM EtherCAT, Synapticon, Germany) via SPI and placed on the rigid link next to the joint of the exoskeleton (Fig. 8).

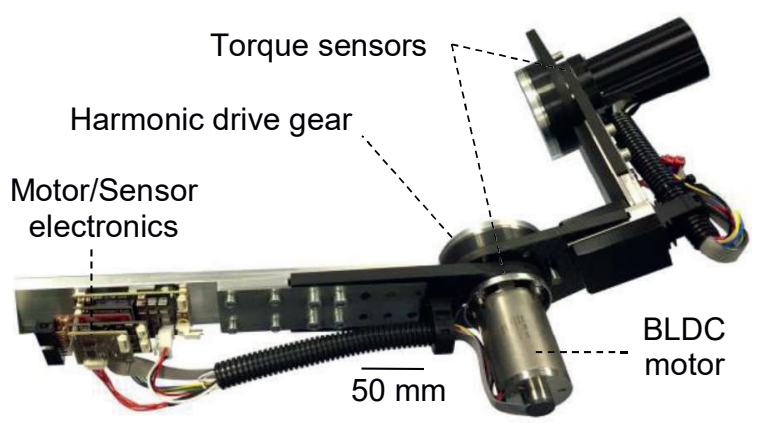

Fig. 8: 2 DOF shoulder exoskeleton with integrated torque sensors and electronics.

When driving the motor with a position controlled sinus joint angle, a repeatable stick-slip effect is noticeable (Fig. 9). The error is caused by friction in the gear drive and must be considered in joint torque computations.

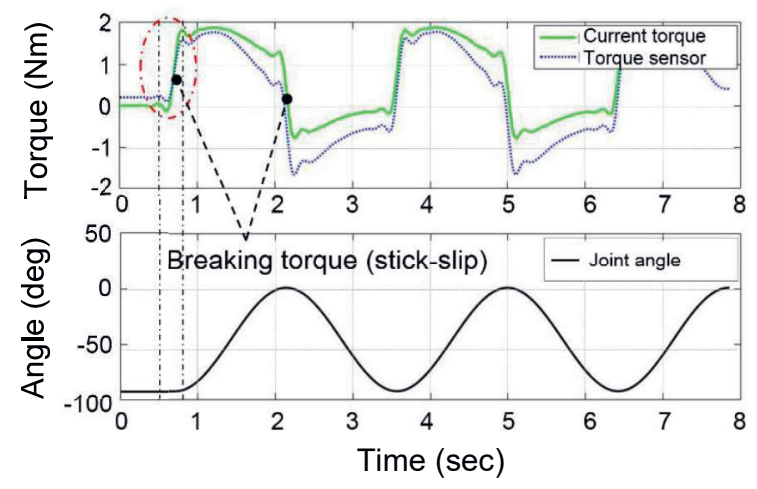

Fig. 9: Measurement results of the integrated torque sensor compared to the resulting joint angle and motor current torque. Repeatable stick-slip friction effect due to position control.

The sensor provides reliable measurement values of the joint torque. Oscillatory instabilities and offset errors are reduced compared to torque estimation via motor current during impedance control.

\section{User interaction measurement}

The integrated torque sensor measures the sum of torques created by the motor, dynamics of the exoskeleton, the user and interaction with the environment. In order to estimate the user interaction torques with the exoskeleton to derive the user's movement intention, it is necessary to subtract the other applied torques from the measured torque $\tau_{s}$ (Fig. 10). 


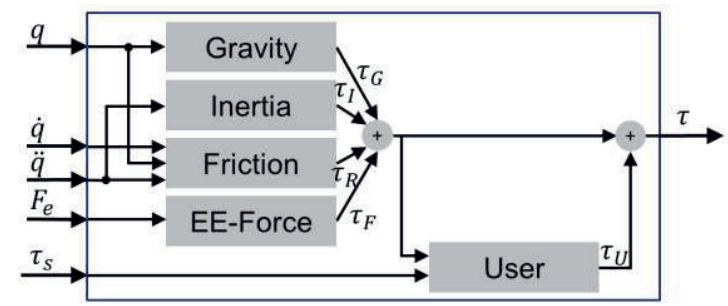

Fig. 10: Dynamic influences on torque measurement.

Therefore, the desired torque of the exoskeleton

$$
\tau=\tau_{G}(q)+\tau_{I}(\ddot{q})+\tau_{R}(q, \dot{q}, \ddot{q})+\tau_{F}\left(F_{e}\right)+\tau_{U}\left(\tau_{s}\right)
$$

is the sum of torques caused by gravity $\tau_{G}$, inertia $\tau_{I}$, friction $\tau_{R}$ [13], the end effector (EE) interaction $\tau_{F}$ [14] and the user interaction $\tau_{U}$.

If the user is not applying any torque to the system, the torque sensor only measures the dynamics and interaction with the environment (Fig. 11, blue curve). If the user is applying a torque to the joints of the exoskeleton during the same operation, the measured torque increases (Fig. 11, green curve).

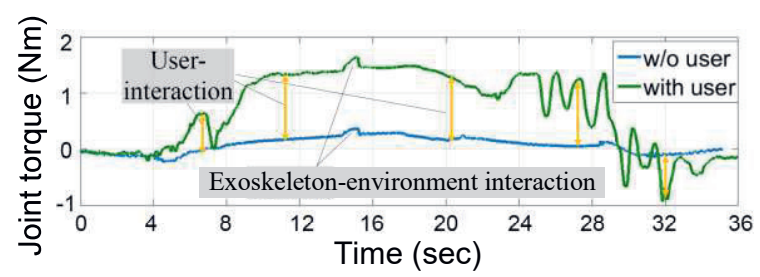

Fig. 11: Joint torque measurement during positioning tasks of the exoskeleton and interaction with the environment (position controlled).

\section{Conclusion}

Measuring joint torque in an exoskeleton is a challenging task. Torque measurement in the joints of the kinematic chain is not possible with regular foil strain gauges due to high transverse forces. Therefore, the sensing element is integrated between the motor and gear of each joint in order to reduce the influence of shear forces and torques. The designed sensor shows excellent transmission behavior for torque measurements in the robotic joints. Thus, movement intentions, applied through joint torques by the user, are detected with the sensor system without using motor current or EMG.

\section{Outlook}

The measurement results show a strong impact of friction in the gear drive to the sensor signal. In the future, a dynamic model for friction in the gear drive will be created and evaluated with measurement data from different gear drives.

\section{References}

[1] P. Rashidi and A. Mihailidis, "A Survey on Ambient-Assisted Living Tools for Older Adults," in IEEE Journal of Biomedical and Health Informatics, vol. 17, no. 3, pp. 579-590, 2013.

[2] K. Kiguchi and H. Yoshiaki, "An EMG-based control for an upper-limb power-assist exoskeleton robot," in IEEE Transactions on Systems, Man, and Cybernetics, Part B (Cybernetics) vol. 42.4, pp. 1064-1071, 2012.

[3] S. Charoenseang and S. Panjan, "Universal Exoskeleton Arm Design for Rehabilitation," in Journal of Automation and Control Engineering Vol 3.6, 2015.

[4] P. Letier et al., "SAM : A 7-DOF portable arm exoskeleton with local joint control," in IEEE/RSJ International Conference on Intelligent Robots and Systems, pp. 3501-3506, 2008.

[5] C. Carignan and M. Liszka, "Design of an arm exoskeleton with scapula motion for shoulder rehabilitation," in IEEE 12th International Conference on Advanced Robotics (ICAR'05), 2005.

[6] R. Steger, S. H. Kim and H. Kazerooni, "Control scheme and networked control architecture for the Berkeley lower extremity exoskeleton (BLEEX)." in IEEE International Conference on Robotics and Automation (ICRA), 2006.

[7] A. Zoss and H. Kazerooni, "Design of an electrically actuated lower extremity exoskeleton." in Advanced Robotics, vol. 20.9, pp. 967-988, 2006.

[8] R. Gopura, K. Kazuo and L. Yang, "SUEFUL-7: A 7DOF upper-limb exoskeleton robot with musclemodel-oriented EMG-based control," in IEEE/RSJ International Conference on Intelligent Robots and Systems (IROS), 2009.

[9] T. Lalitharatne et al., "Evaluation of perceptionassist with an upper-limb power-assist exoskeleton using EMG and EEG signals," in IEEE 11th International Conference on Networking, Sensing and Control (ICNSC), 2014.

[10] B-C. Tsai et al. "An articulated rehabilitation robot for upper limb physiotherapy and training." in IEEE/RSJ International Conference on Intelligent Robots and Systems (IROS), 2010.

[11] K. Kiguchi and Q. Qilong, "Muscle-model-oriented EMG-based control of an upper-limb power-assist exoskeleton with a neuro-fuzzy modifier." in IEEE International Conference on.Fuzzy Systems, 2008.

[12] A. Albu-Schäffer et al., "The DLR Lightweight Robot - Design and Control Concepts for Robots in Human Environments," in Industrial Robot: An International Journal, vol. 34 (5), pp. 376-385, 2008. 
[13] M. Hessinger, N. Faul, P. P. Pott, R.

Werthschützky, "Dynamic Model of an Upper

Limb Exoskeleton with User Interaction," in

Dreiländertagung - Swiss, Austrian and German

Societies of Biomedical Engineering (BMT),

2016.

[14] M. Hessinger, J. Hielscher, P. P. Pott, R. Werthschützky, "Handheld surgical drill with integrated thrust force recognition," in IEEE International Conference on e-Health and Bioengineering (EHB), 2013. 